\title{
Active balancing control for time-delay rotor via linear-quadratic regulator
}

\author{
Juan Xu๑ ${ }^{\text {a,b }}$ Yang Zhao, ${ }^{\mathrm{c}}$ Zhanfeng $\mathrm{Xu},{ }^{\mathrm{c}}$ and Benhong Zhang ${ }^{\mathrm{a}, *}$ \\ ${ }^{a}$ Hefei University of Technology, School of Computer Science and Information Engineering, \\ Hefei, China \\ ${ }^{\mathrm{b}}$ Anhui Fuhuang Technology Co., Ltd., Hefei, China \\ ${ }^{c}$ Hefei University of Technology, School of Mechanical Engineering, Hefei, China
}

\begin{abstract}
Many imaging platforms, e.g., robotic devices and autonomous systems, require timely reaction to external events. The active balancing control is important to suppress rotor vibration and hence acquire stable videos and imagery. However, there is an inevitable delay in active balancing control of the high-speed, time-delay rotor systems. Eliminating this delay is crucial in real-world applications. The integral transformation term is introduced to transform the dynamic model of the time-delay rotor system into a dynamic model of the rotor system without time delay. Based on the linear-quadratic optimal control method, the influence of weight matrix is discussed. Moreover, an active balancing control strategy of the time-delay rotor is designed. The simulation model of the time-delay rotor control system is implemented with MATLAB, and a real device is fabricated to conduct both simulations and real-world experiments. Our results demonstrate that our proposed online active balancing control method effectively suppresses the vibration of the time-delay rotor system under different time-delay. The convergence time is short, and the system is agile. (c) The Authors. Published by SPIE under a Creative Commons Attribution 4.0 Unported License. Distribution or reproduction of this work in whole or in part requires full attribution of the original publication, including its DOI. [DOI: 10.1117/1.JEI.30.3.031205]
\end{abstract}

Keywords: active balancing control; time-delay; vibration; linear quadratic optimization.

Paper 200551SS received Aug. 17, 2020; accepted for publication Nov. 16, 2020; published online Jan. 21, 2021.

\section{Introduction}

At present, many imaging systems require the ability to interact with external events in real time. Robot equipment is widely used in various imaging systems. Therefore, the vibration of equipment will seriously affect the stability of imaging systems. ${ }^{1,2}$ The active balancing control can reduce the vibration of the rotor system without stopping the rotor. ${ }^{3,4}$ The traditional active balancing control method often ignores the time delay in the balancing process. In fact, the slight time delay will also lead to the reduction of the control efficiency during the automatic balancing process. ${ }^{5,6}$ Seriously, the time-delay rotor system may be unstable and cause a major accident. ${ }^{7,8}$ Therefore, it is of great significance to take the time-delay into account in the design of active balancing control of the rotor system.

Domestic and foreign scholars have done a lot of research on online dynamic balance of rotor systems with time delay. Zheng et al. ${ }^{9}$ studied the active longitudinal vibration control, derived the frequency response function of the disturbance and control channels using the quadrupole parameter method, and analyzed the active control effect by numerical simulation. Saeed and ElGanaini ${ }^{10}$ employed the nonlinear time-delay position-velocity feedback controller to control the system lateral vibrations and showed how to harness the time-delays to reduce the oscillations of the system without affecting its stability. Wang et al. ${ }^{11}$ accomplished the uncertainty quantification analysis under aleatory and epistemic uncertainties and used a new hybrid time-variant reliability index to judge the safety levels for controlled structures. $\mathrm{Hu}$ and $\mathrm{He}^{12}$ designed a new rotor dynamic vibration absorber and applied an on-off control method based on speed to reduce the vibration in time-delay rotor systems. A PID semiactive control algorithm was designed by Huang et al. ${ }^{13}$ to control the suspension system, and the design method of multi-time-delay

*Address all correspondence to Benhong Zhang, zhangbh@hfut.edu.cn 
control law for active structural vibration control was proposed, which is easily realized in practical application and performs with strong robustness. Zhang et al. ${ }^{14}$ proposed a robust predictive control model for positive delay systems with uncertainty and interval uncertainty.

However, the feedback control of time-delay systems is mainly applied in the structural vibration. It is very difficult to identify the stability characteristics of complex time-varying systems and achieve accurate solutions for nonlinear multi-degree-of-freedom systems with time-delay. In fact, the time-delay problem in the rotor system cannot be ignored in the design of control strategies. Thus, this paper proposed an active balancing control method for a timedelay rotor based on LQR control, and the model is verified through a series of experiments.

The main contribution of this work is twofold:

1. Different from the existing methods that focus on the complicated modeling and solving the time-delay nonlinear systems, this paper proposes an idea to transform the time-delay rotor system model into a rotor subsystem model without time-delay by introducing an integral transformation term. The challenge of solving the time-varying nonlinear multi-degree-of-freedom dynamics system can be transformed into the control law design problem of the state space equation.

2. In order to overcome the drawback of finite-time optimal control, we define the linearquadratic optimal performance index function of the system, and the linear-quadratic optimal control strategy of infinite time is designed using the optimal control theory. The active balancing control system has the ability to maintain the balanced state in infinite time.

\section{Proposed Method}

\subsection{System Model}

The rotor active balancing control system generally includes a rotating speed sensor, a vibration sensor, an active balance controller, and an actuator, as shown in Fig. 1. The vibration sensor and the rotating speed sensor acquire the vibration signal caused by the rotor imbalance and the rotating speed signal of the rotor and transmit the signal to the active balance controller. ${ }^{15}$ The active balance controller analyzes and processes the input signal to calculate the control output signal, which can drive the actuator to suppress the vibration caused by the imbalance.

The dynamic model of the time-delay rotor system is established, as shown in Fig. 2. It consists of a rotating shaft, rigid discs, and bearings that do not take into account mass. The mass of the rotor disc is $m$, the stiffness of the rotating shaft at the center point is $k$, the damping coefficient of the time-delay rotor system is $c$, and the rotating angular velocity of the time-delay rotor system is $\omega$. The rotating angle is $\alpha$. Assuming that $S$ point is the geometric center of the disk, the axis of rotation passes through the geometric center of the disk, and the center of mass $G$ of the rotor deviates from the geometric center of the rotor, resulting in an eccentricity of $S$.

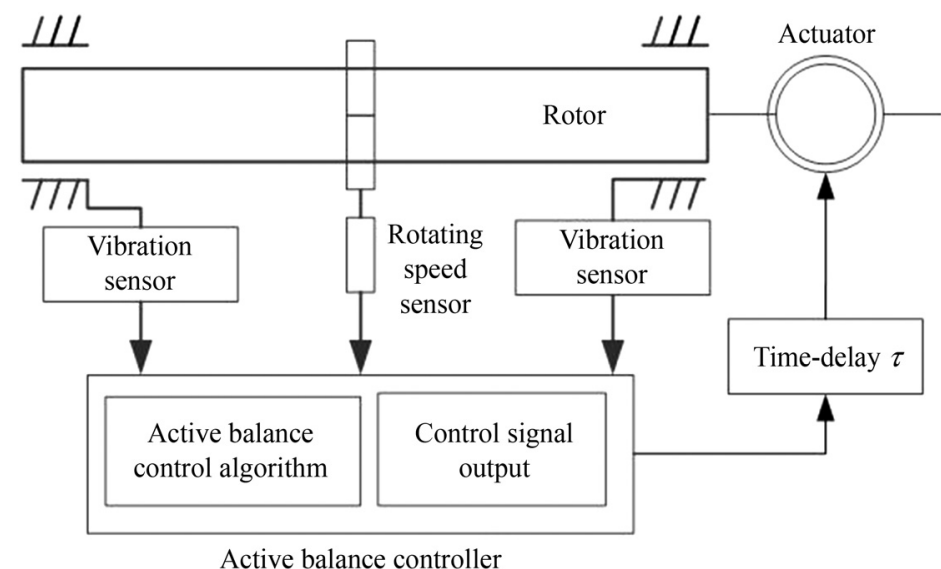

Fig. 1 The schematic of active balancing control system. 

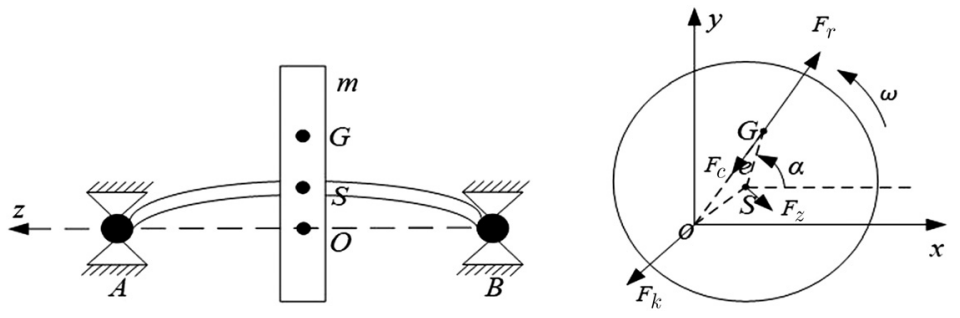

Fig. 2 Schematic diagram of rotor structure.

The line connecting the $A B$ lines is the $z$ axis, and the disc is at $O$ points. The centrifugal force of the disc caused by imbalance is $F_{r}$, the elastic restoring force of the elastic shaft is $F_{k}$, and the damping force $F_{z}$ is opposite to the moving direction of the rotor. The damping force is proportional to the absolute speed of the rotor. The space orthogonal coordinate system of the time-delay rotor system is established by taking the straight line of the initial position of $F_{c}$ as the $x$ axis and the $y$ axis simultaneously perpendicular to the $x$ axis and the $z$ axis.

When the time-delay rotor system rotates at an angular velocity $\omega$, the dynamic equations of the rotor in both directions of $x$ axis and $y$ axis are as follows:

$$
\left\{\begin{array}{l}
m \ddot{x}_{G}+k x+c \dot{x}=0 \\
m \ddot{y}_{G}+k y+c \dot{y}=0
\end{array},\right.
$$

where $m \ddot{x}_{G}$ and $m \ddot{y}_{G}$ are the centrifugal force component generated on the $x$ axis and $y$ axis respectively in the process of rotor rotation.

If the coordinate of $G$ point is $G\left(x_{G}, y_{G}\right)$, the coordinates of $S$ point is $S(x, y)$, and the coordinate relationship between the disc centroid $G$ and the geometric center $S$ can be obtained:

$$
\left\{\begin{array}{l}
x_{G}=x+e \cos \alpha \\
y_{G}=y+e \sin \alpha
\end{array} .\right.
$$

By taking Eq. (2) into Eq. (1), it can be written as

$$
\left\{\begin{array}{l}
m \ddot{x}+c \dot{x}+k x=m e\left(\dot{\alpha}^{2} \cos \alpha+\ddot{\alpha} \sin \alpha\right) \\
m \ddot{y}+c \dot{y}+k y=m e\left(\dot{\alpha}^{2} \sin \alpha-\ddot{\alpha} \cos \alpha\right)
\end{array},\right.
$$

when the rotor runs at a steady speed, $\ddot{\alpha}=0, \dot{\alpha}=\omega$, and $\alpha=\omega t$, Eq. (3) can be simplified as

$$
\left\{\begin{array}{l}
m \ddot{x}+c \dot{x}+k x=m e \omega^{2} \cos \omega t \\
m \ddot{y}+c \dot{y}+k y=m e \omega^{2} \sin \omega t
\end{array} .\right.
$$

The above dynamics [Eq. (4)] of the time-delay rotor system can be represented in a matrix form:

$$
M \ddot{X}+C \dot{X}+K X=F_{r},
$$

where the mass matrix $M=\left(\begin{array}{cc}m & 0 \\ 0 & m\end{array}\right)$, damping matrix $C=\left(\begin{array}{ll}c & 0 \\ 0 & c\end{array}\right)$, stiffness matrix $K=\left(\begin{array}{ll}k & 0 \\ 0 & k\end{array}\right)$, centrifugal force vector $F_{r}=\left(\begin{array}{cc}m e \omega^{2} & \cos \omega t \\ m e \omega^{2} & \sin \omega t\end{array}\right)$, and vibration of rotor $X=\left(\begin{array}{l}x \\ y\end{array}\right)$.

During the active balancing control of the rotor, the controller drives the actuator to generate an active balancing control force $F_{c}$ that acts on the time-delay rotor system:

$$
M \ddot{X}+C \dot{X}+K X=F_{r}+F_{c} .
$$

Due to the inevitable time-delay $\tau$ of the control signal transmitted to the actuator process, the active balance control force with time-delay is introduced into the dynamic equation of the time-delay rotor system: 


$$
M \ddot{X}+C \dot{X}+K X=F_{r}(t)+F_{c}(t-\tau) .
$$

Equation (7) is equivalent to Eq. (8)

$$
\ddot{X}+M^{-1} C \dot{X}+M^{-1} K X=M^{-1} F_{r}(t)+M^{-1} F_{c}(t-\tau) .
$$

Convert Eq. (8) to state space, the state space equation can be expressed as

$$
\left\{\begin{array}{l}
\dot{Z}(t)=A Z(t)+B_{1} u(t-\tau)+B_{p} u_{p}(t) \\
Y(t)=D Z(t)
\end{array}\right.
$$

where $Z(t)=\left(\begin{array}{l}X(t) \\ \dot{X}(t)\end{array}\right)=\left(\begin{array}{llll}x & y & \dot{x} & \dot{y}\end{array}\right)^{\mathrm{T}}, A=\left(\begin{array}{cc}0 & I \\ -M^{-1} K & -M^{-1} C\end{array}\right), B_{1}=B_{p}=\left(\begin{array}{c}0 \\ M^{-1}\end{array}\right)$, $u(t-\tau)=F_{c}(t-\tau), u_{p}(t)=F_{r}(t), D$ is the output matrix, and $Y(t)$ is the output.

\subsection{Transformation of Dynamic Model of Time-Delay Rotor System}

Since the state equation of the time-delay rotor system contains time-delay $\tau$, in the rotor active balancing control process, the active balancing controller will input energy to the time-delay rotor system when the system does not need energy. That will reduce the efficiency of active balancing control and even lead to the instability of the rotor system. ${ }^{16,17}$ Considering that it is relatively difficult to directly design the control law of the time-delay rotor system, to eliminate the influence of time-delay $\tau$ on the active balance control system, the following transformation is introduced: ${ }^{18}$

$$
E(t)=Z(t)+\Gamma(t)
$$

where $\Gamma(t)$ represents the integral term associated with $\tau$

$$
\Gamma(t)=\int_{t-\tau}^{t} e^{-A(s-t)} e^{-A \tau} B_{1} u(s) \mathrm{d} s .
$$

Deriving Eq. (10)

$$
\begin{aligned}
\dot{E}(t) & =\dot{Z}(t)+\left[\int_{t-\tau}^{t} e^{-A(s-t)} e^{-A \tau} B_{1} u(s) \mathrm{d} s\right]^{\prime} \\
& =\dot{Z}(t)+\left[\int_{0}^{t} e^{-A(s-t)} e^{-A \tau} B_{1} u(s) \mathrm{d} s\right]^{\prime}-\left[\int_{0}^{t-\tau} e^{-A(s-t)} e^{-A \tau} B_{1} u(s) \mathrm{d} s\right]^{\prime} .
\end{aligned}
$$

In Eq. (11), we solve

$$
\begin{aligned}
{\left[\int_{0}^{t} e^{-A(s-t)} e^{-A \tau} B_{1} u(s) \mathrm{d} s\right]^{\prime} } & =\lim _{\Delta t \rightarrow 0} \frac{\int_{0}^{t+\Delta t} e^{-A(s-t-\Delta t)} e^{-A \tau} B_{1} u(s) \mathrm{d} s-\int_{0}^{t} e^{-A(s-t)} e^{-A \tau} B_{1} u(s) \mathrm{d} s}{\Delta t} \\
& =A \int_{0}^{t} e^{-A(s-t)} e^{-A \tau} B_{1} u(s) \mathrm{d} s+e^{-A \tau} B_{1} u(t)
\end{aligned}
$$

Similarly,

$$
\begin{aligned}
& {\left[\int_{0}^{t-\tau} e^{-A(s-t)} e^{-A \tau} B_{1} u(s) \mathrm{d} s\right]^{\prime}} \\
& \quad=\lim _{\Delta t \rightarrow 0} \frac{\int_{0}^{t-\tau+\Delta t} e^{-A(s-t-\Delta t)} e^{-A \tau} B_{1} u(s) \mathrm{d} s-\int_{0}^{t-\tau} e^{-A(s-t)} e^{-A \tau} B_{1} u(s) \mathrm{d} s}{\Delta t} \\
& \quad=A \int_{0}^{t-\tau} e^{-A(s-t)} e^{-A \tau} B_{1} u(s) \mathrm{d} s+B_{1} u(t-\tau)
\end{aligned}
$$


Substituting Eqs. (12) and (13) into Eq. (11) for simplification

$$
\begin{aligned}
\dot{E}(t) & =\dot{Z}(t)+\left[\int_{t-\tau}^{t} e^{-A(s-t)} e^{-A \tau} B_{1} u(s) \mathrm{d} s\right]^{\prime} \\
& =\dot{Z}(t)+A \int_{t-\tau}^{t} e^{-A(s-t)} e^{-A \tau} B_{1} u(s) \mathrm{d} s+e^{-A \tau} B_{1} u(t)-B_{1} u(t-\tau) .
\end{aligned}
$$

Combining the state space [Eq. (9)], substituting Eq. (9) into Eq. (14) for simplification

$$
\dot{E}(t)=A E(t)+B_{p} u_{p}(t)+e^{-A \tau} B_{1} u(t) .
$$

Finally, the state space equation of the time-delay rotor system without time-delay can be obtained as

$$
\left\{\begin{array}{l}
\dot{E}(t)=A E(t)+B u(t)+B_{p} u_{p}(t) \\
H(t)=D E(t)
\end{array}\right.
$$

where $E(t)=(\hat{x}, \hat{y}, \hat{\dot{x}}, \hat{\dot{y}})^{T}$ is the state variable of the transformed time-delay rotor system, $H(t)$ is the output vector of the time-delay rotor system, $A=\left(\begin{array}{cc}0 & I \\ -M^{-1} K & -M^{-1} C\end{array}\right), B=e^{-A \tau} B_{1}$, $B_{p}=\left(\begin{array}{c}0 \\ M^{-1}\end{array}\right)$, and $D$ is the output matrix of the control system.

\subsection{Design of LQR Active Balancing Control Law}

The optimal control algorithm is widely used in the field of active vibration control. ${ }^{19,20}$ Linear quadratic control can obtain the optimal control law of state linear feedback to realize closedloop optimal control. Furthermore, linear quadratic control can keep the error close to zero with the minimum energy cost. Therefore, we designed the active balancing control law for timedelay rotors using the linear-quadratic optimal control theory. According to the theory of optimal control, the external excitation term of the time-delay rotor system can be ignored at first. When designing the optimal control law, we introduced a performance index of optimal control. The linear-quadratic optimal performance index function of the system is given:

$$
J=\frac{1}{2} \int_{t_{0}}^{t_{1}}\left[E^{\mathrm{T}}(t) Q E(t)+u^{\mathrm{T}}(t) R u(t)\right]
$$

where $Q$ and $R$ are the positive definite gain matrix of the state variable and the semi-positive definite gain matrix of the input variable, $t_{0}$ and $t_{1}$ are the start time and the end time of active balancing control, respectively. We set $t_{0}=0, t_{1}=+\infty$.

The optimal control is to solve the optimal control law $u(t)$. The design of the state feedback controller $G$ is to minimize the performance index function $J$ of the linear-quadratic optimal control for the given system. The linear-quadratic optimal control law is obtained as

$$
u(t)=-G E(t) .
$$

Among them, $G=R^{-1} B^{T} P(t), p(t)$ is the solution of Riccati equation. Riccati equation is expressed as

$$
-P A-A^{T} P+P B R^{-1} B^{T} P-Q=0 .
$$

The Riccati Eq. (19) is to obtain the feedback matrix $G$ of the controller. The block diagram of the LQR control system of the time-delay rotor is shown in Fig. 3.

Thereby, the key to designing the linear quadratic optimal control law is to select the appropriate weight matrix $Q$ and $R$. When the weight matrix $Q$ increases, the control rate of the system increases, the control time to reach stability decreases, but the steady-state error of the control system increases. When the weight matrix $R$ increases, the control quantity of the system 


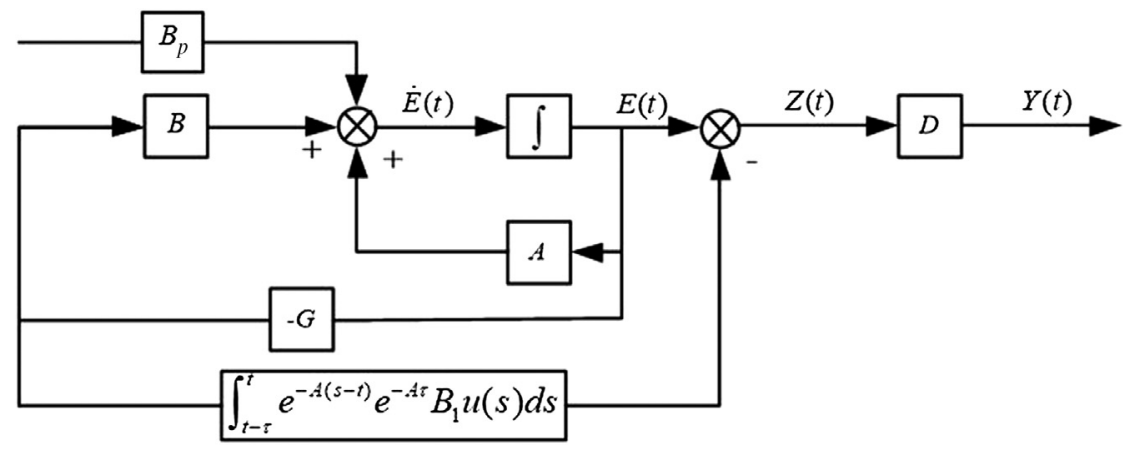

Fig. 3 LQR control system block diagram of a time-delay rotor.

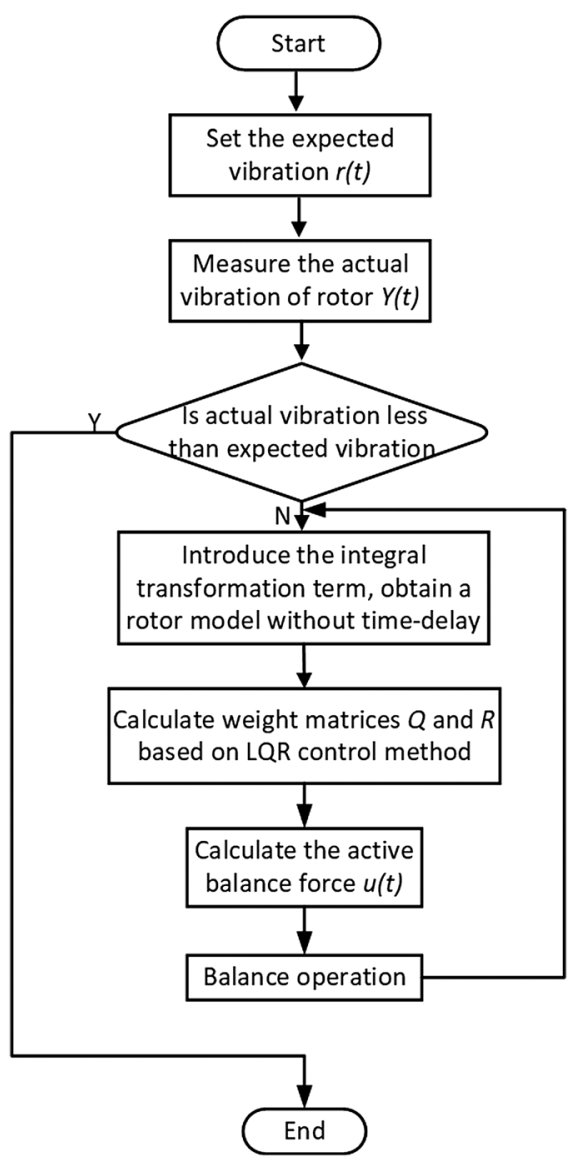

Fig. 4 Flowchart of the control algorithm.

decreases, and the control time of the system increases. In this paper, the influence of different weight matrices $Q$ and $R$ on the control system will be verified by simulation, and a linear quadratic optimal control law is designed by choosing appropriate weight matrices $Q$ and $R$. The flowchart of the algorithm is shown in Fig. 4.

\section{Results and Discussion}

\subsection{System Simulation Model}

To evaluate our proposed online active balancing control method for the time-delay rotors, we conducted simulation experiments. The method proposed in this paper is mainly aimed at the medium-high speed rotor system. In our experiment, the rotor speed is set to $3000 \mathrm{rpm}$, 
Table 1 Parameters of the time-delay rotor system.

\begin{tabular}{lc}
\hline \hline Parameter & Value \\
\hline Disc quality & $m=1.5 \mathrm{~kg}$ \\
Bending stiffness & $k=2000 \mathrm{~N} / \mathrm{m}$ \\
Damping coefficient & $c=0.2 \mathrm{Ns} / \mathrm{m}$ \\
Eccentricity & $e=0.002 \mathrm{~m}$ \\
Rotor speed & $n=3000 \mathrm{rpm}$ \\
Time-delay & $\tau=0.001 \mathrm{~s}$ or $0.01 \mathrm{~s}$ \\
\hline \hline
\end{tabular}

other parameters are taken from empirical values to more accurately simulate the actual working conditions. The parameters of the rotor system are shown in Table 1.

According to the parameters of the above time-delay rotor system takes $\tau=0.01 \mathrm{~s}$ as an example, the parameter matrix in the state space equation of the time-time-delay rotor system can be obtained, as shown below:

$$
\begin{gathered}
A=\left(\begin{array}{cccc}
0 & 0 & 1 & 0 \\
0 & 0 & 0 & 1 \\
-1333.333 & 0 & -0.1333 & 0 \\
0 & -1333.333 & 0 & -0.1333
\end{array}\right), \quad B=\left(\begin{array}{cc}
-0.0007 & 0 \\
0 & -0.0007 \\
0.6663 & 0 \\
0 & 0.6663
\end{array}\right), \\
B_{p}=\left(\begin{array}{cc}
0 & 0 \\
0.6667 & 0 \\
0 & 0.6667
\end{array}\right), \quad D=\left(\begin{array}{llll}
1 & 0 & 0 & 0 \\
0 & 1 & 0 & 0
\end{array}\right) .
\end{gathered}
$$

Based on the state space equation of the original time-delay rotor system, the integral transformation method is introduced to calculate the state space equation without time-delay. Since the vibration of the time-delay rotor system in the $x$-axis and $y$-axis directions is similar, we focus on the vibration in the $x$-axis direction, and the simulation model of the active balancing control of the time-delay rotor system is established, as shown in Fig. 5.

\subsection{Step Response}

Generally speaking, for the control system, step input is the most severe working state of the control system. If the control system can satisfy the control performance requirement under the action of step input, the control system can still satisfy the control performance requirement under the action of other signals. Thereby we select the step input as the input signal in the case of $\tau=0.001 \mathrm{~s}$ for the simulation experiment. In order to study the influence of weight matrix $Q$ and $R$ on the active balancing control system of time-delay rotor, we select different weight matrix $Q$ and $R$ and calculate the state feedback matrix $G$, then the step response of the control system is obtained. The open-loop step response of the time-delay rotor system is shown in Fig. 6.

The weight matrix in this paper is selected separately

$$
Q=\alpha\left(\begin{array}{cc}
K & 0 \\
0 & M
\end{array}\right)=\alpha\left(\begin{array}{cccc}
2000 & 0 & 0 & 0 \\
0 & 2000 & 0 & 0 \\
0 & 0 & 1.5 & 0 \\
0 & 0 & 0 & 1.5
\end{array}\right), \quad R=\beta\left(\begin{array}{ll}
1 & 0 \\
0 & 1
\end{array}\right)
$$




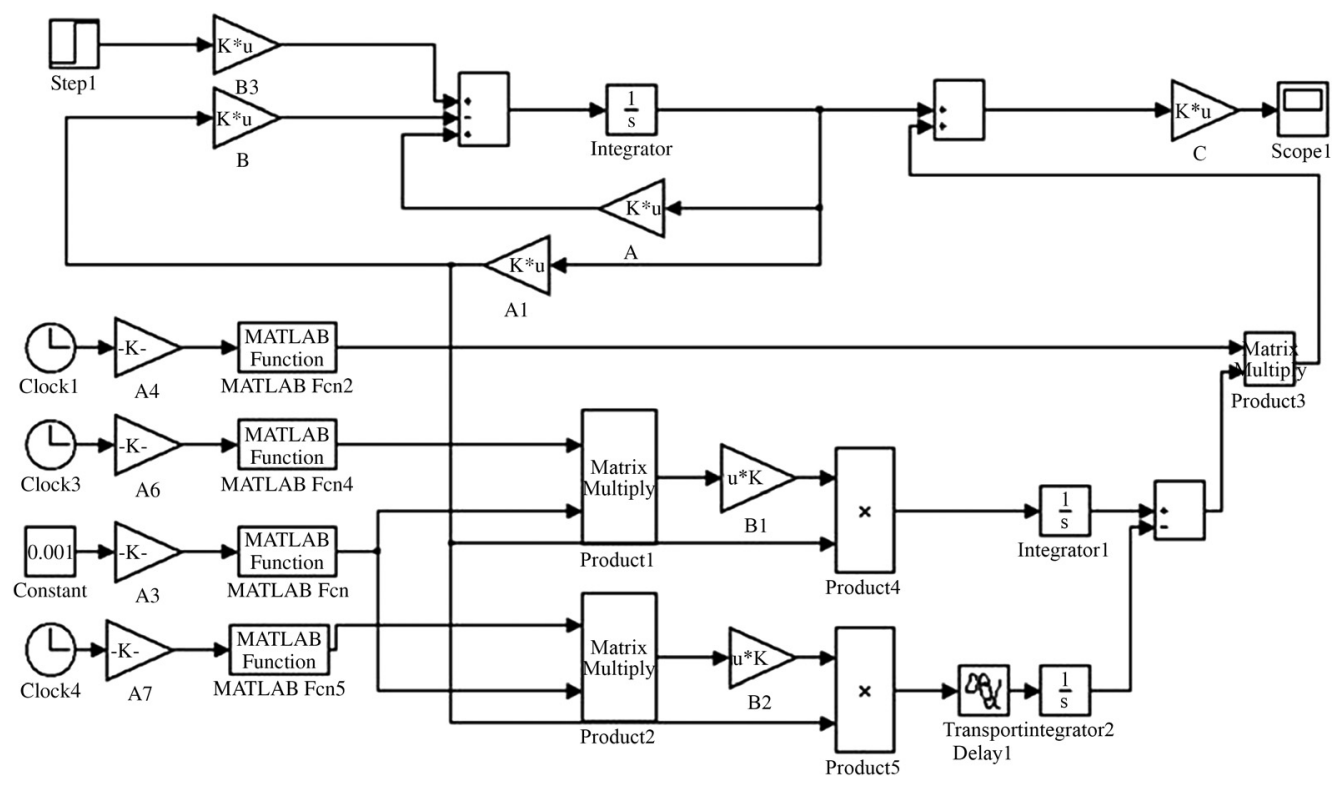

Fig. 5 Simulation model of active balance control of time-delay rotor system.

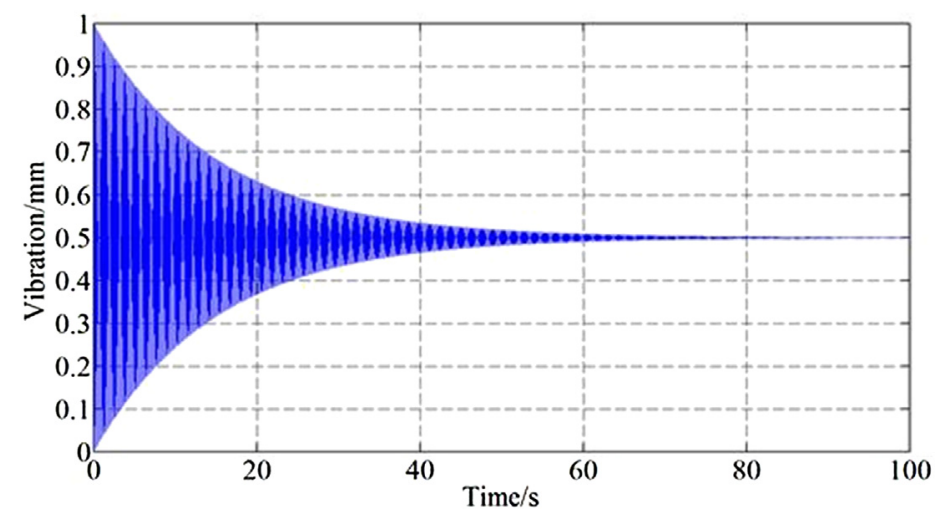

Fig. 6 Open-loop step response of time-delay rotor system.

where $\alpha$ and $\beta$ are undetermined coefficients. Assuming that the weight matrix $R$ is invariant, the influence of the weight matrix $Q$ of the rotor control system is discussed. Figure 7 shows the step response of the time-delay rotor system when $\alpha$ is $0.1,1,10$, and 100, respectively.

Assuming that the weight matrix $Q$ is invariant, the influence of the weight matrix $R$ of the rotor control system is discussed. Figure 8 shows the step response of the time-delay rotor system when $\beta$ is $0.1,1,10$, and 100 , respectively.

As shown in Figs. 7 and 8, when the weight matrix $Q$ is large, the time for the control system to reach stability decreases, but the steady-state error increases. When the weight matrix $R$ is large, the time for the time-delay rotor system to reach stability increases. Thus, we combine the above simulation results about the weight matrix $Q$ and $R$ and carry out several simulation experiments, then the appropriate weight matrix is chosen as below. The control feedback matrix is calculated:

$$
Q=\left(\begin{array}{cccc}
2000 & 0 & 0 & 0 \\
0 & 2000 & 0 & 0 \\
0 & 0 & 1.5 & 0 \\
0 & 0 & 0 & 1.5
\end{array}\right), \quad R=\left(\begin{array}{cc}
0.6 & 0 \\
0 & 0.6
\end{array}\right)
$$




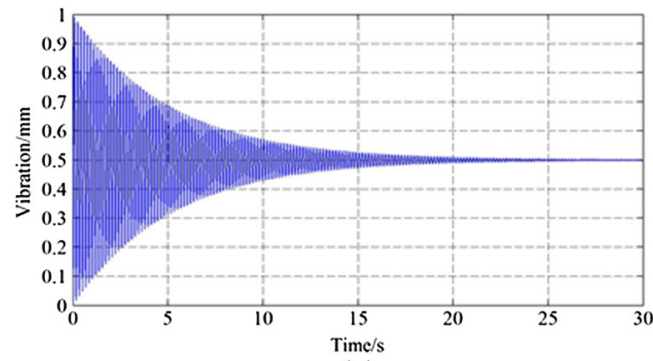

(a)

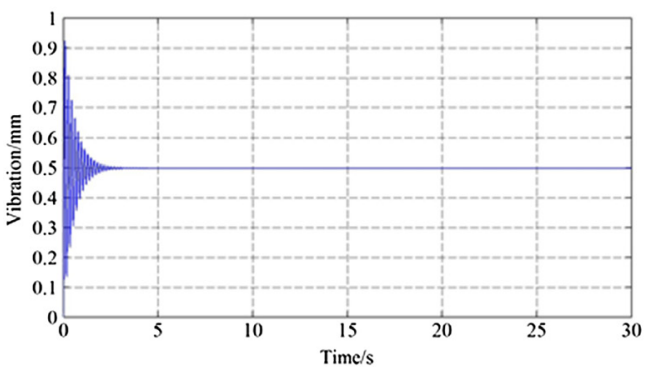

(c)

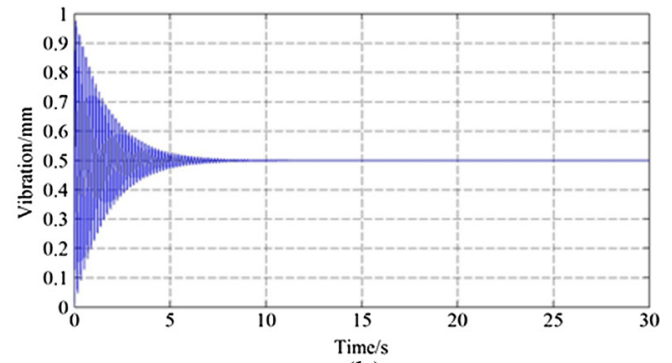

(b)

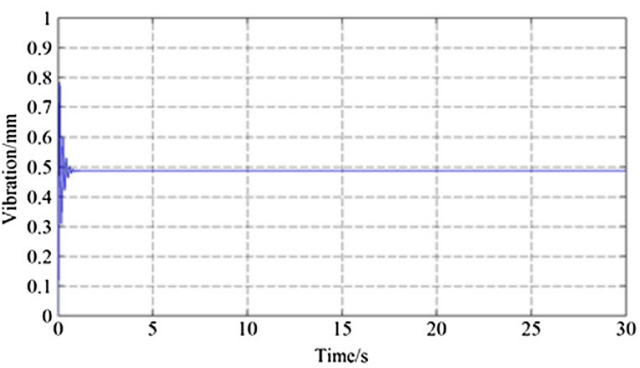

(d)

Fig. 7 Step response of time-delay rotor system with different $\alpha$ when time-delay $\tau=0.001 \mathrm{~s}$.

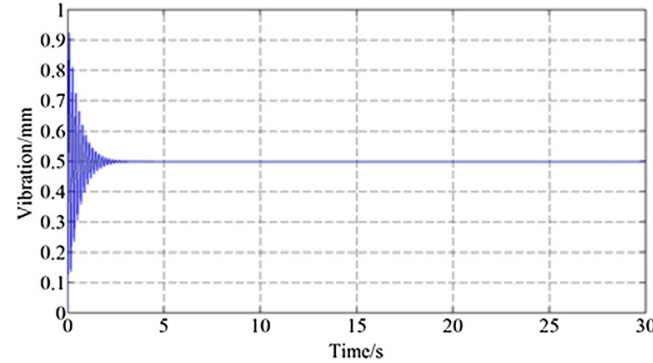

(a)

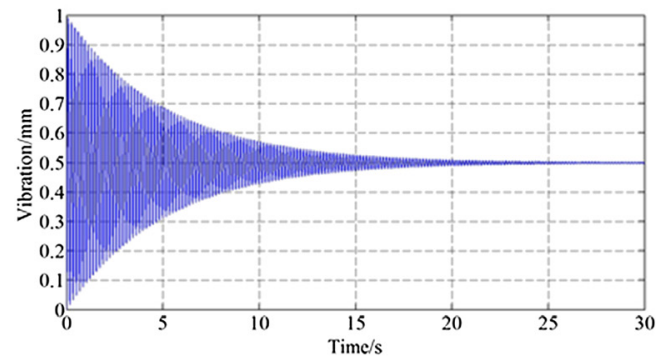

(c)

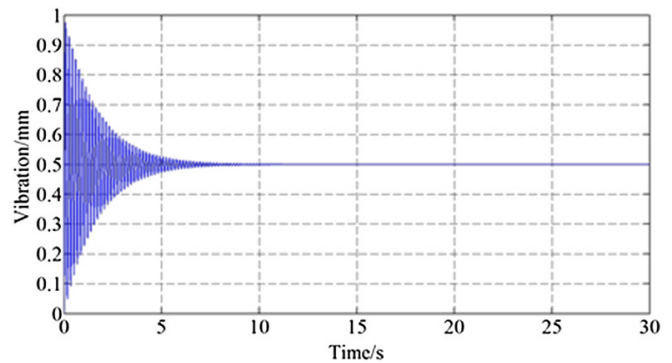

(b)

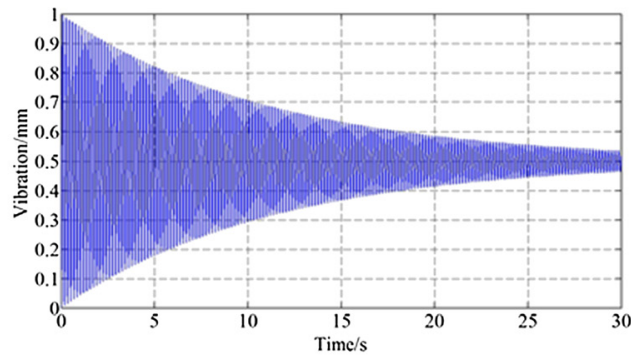

(d)

Fig. 8 Step response of time-delay rotor system with different $\beta$ when time-delay $\tau=0.001 \mathrm{~s}$.

To simulate the actual situation of the time-delay rotor system, we add the centrifugal force into the model. We set the rotational speed $n=3000 \mathrm{~s}$, when the model of the time-delay rotor system without active balancing control force, the time domain figure of the vibration in the $x$-axis direction of the rotor is shown in Fig. 9(a).

Assuming that the rotor system does not contain time-delay, the linear-quadratic optimal control law is introduced to design the linear-quadratic optimal control law of the rotor system, and the simulation results are shown in Fig. 9(b).

Then assuming that the rotor system is with some different time-delay, the linear-quadratic optimal control laws of the time-delay rotor system are calculated. The vibration control process 


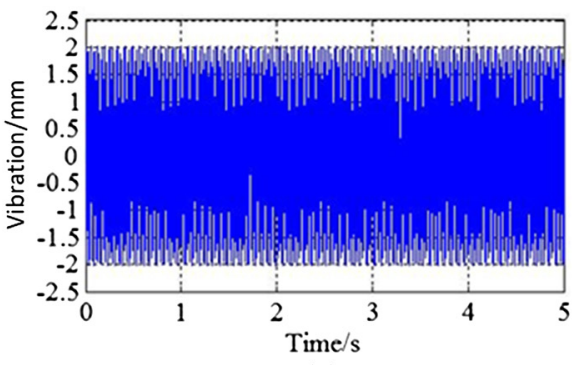

(a)

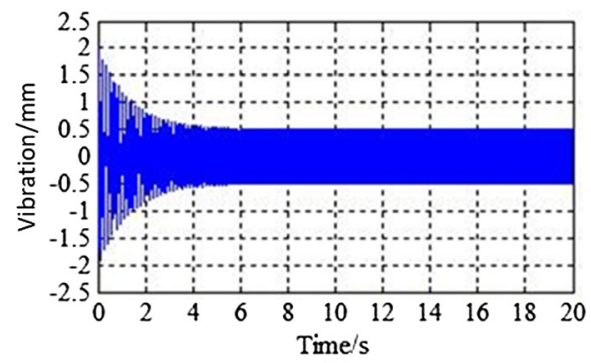

(b)

Fig. 9 Vibration time domain of rotor system.

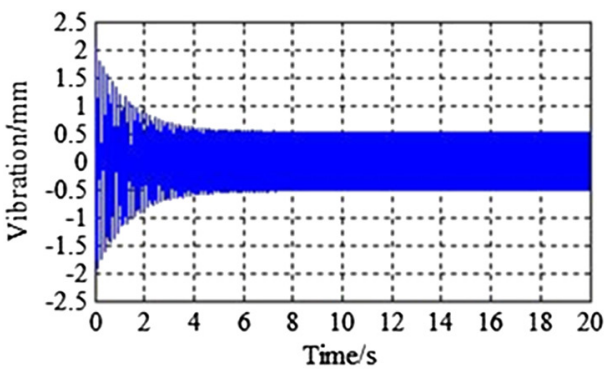

(a)

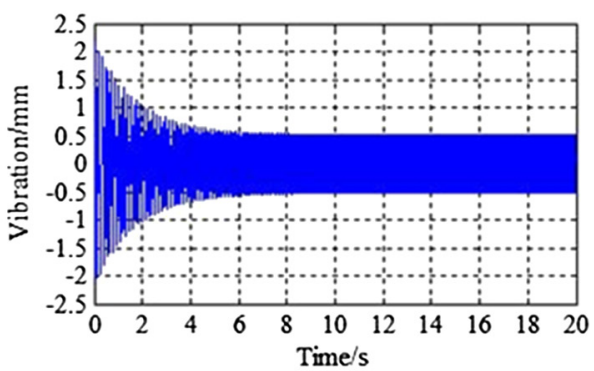

(b)

Fig. 10 Control process of the time-delay rotor system.

Table 2 Comparison of simulation results.

\begin{tabular}{lllc}
\hline \hline $\begin{array}{l}\text { Time- } \\
\text { delay } \\
\text { condition }\end{array}$ & $\begin{array}{c}\text { Initial } \\
\text { vibration }\end{array}$ & $\begin{array}{c}\text { Balanced } \\
\text { vibration }\end{array}$ & $\begin{array}{c}\text { Balancing } \\
\text { time }\end{array}$ \\
\hline$\tau=0 \mathrm{~s}$ & $2.03 \mathrm{~mm}$ & $0.5 \mathrm{~mm}$ & $5.2 \mathrm{~s}$ \\
$\tau=0.1 \mathrm{~s}$ & $2.03 \mathrm{~mm}$ & $0.5 \mathrm{~mm}$ & $6.4 \mathrm{~s}$ \\
$\tau=0.01 \mathrm{~s}$ & $2.03 \mathrm{~mm}$ & $0.5 \mathrm{~mm}$ & $8.5 \mathrm{~s}$ \\
\hline \hline
\end{tabular}

of the time-delay rotor system is obtained when $\tau=0.001 \mathrm{~s}$ and $\tau=0.01 \mathrm{~s}$, as shown in Fig. 10 . The different simulation results with different time-delay conditions are also compared in Table 2.

As can be seen from Table 2, the initial vibration of the time-delay rotor system is $2.03 \mathrm{~mm}$, and the target balanced vibration is $0.5 \mathrm{~mm}$. After active balancing control, when there is no time-delay in the active balancing control process, the time for the control system to reach the balanced state is $5.2 \mathrm{~s}$. When the time-delay is $\tau=0.001 \mathrm{~s}$ in the active balancing control process, the time for the control system to reach the balanced state is $6.4 \mathrm{~s}$. When the time-delay is $\tau=0.01 \mathrm{~s}$ in the rotor active balancing control process, the time is $8.5 \mathrm{~s}$.

Therefore, the linear-quadratic time-delay rotor active balancing control method in this paper can achieve satisfactory vibration control effects under different time-delay. The control effect is similar to that without time delay.

\section{Implementation}

To evaluate the practical utility of the proposed method, a rotor dynamic balancing system is implemented using a variable frequency motor (power $750 \mathrm{~W}$, torque $2.4 \mathrm{NM}$ ), the high-speed spindle, the experimental bearing, the balancing head, and the vibration sensor (eddy current 


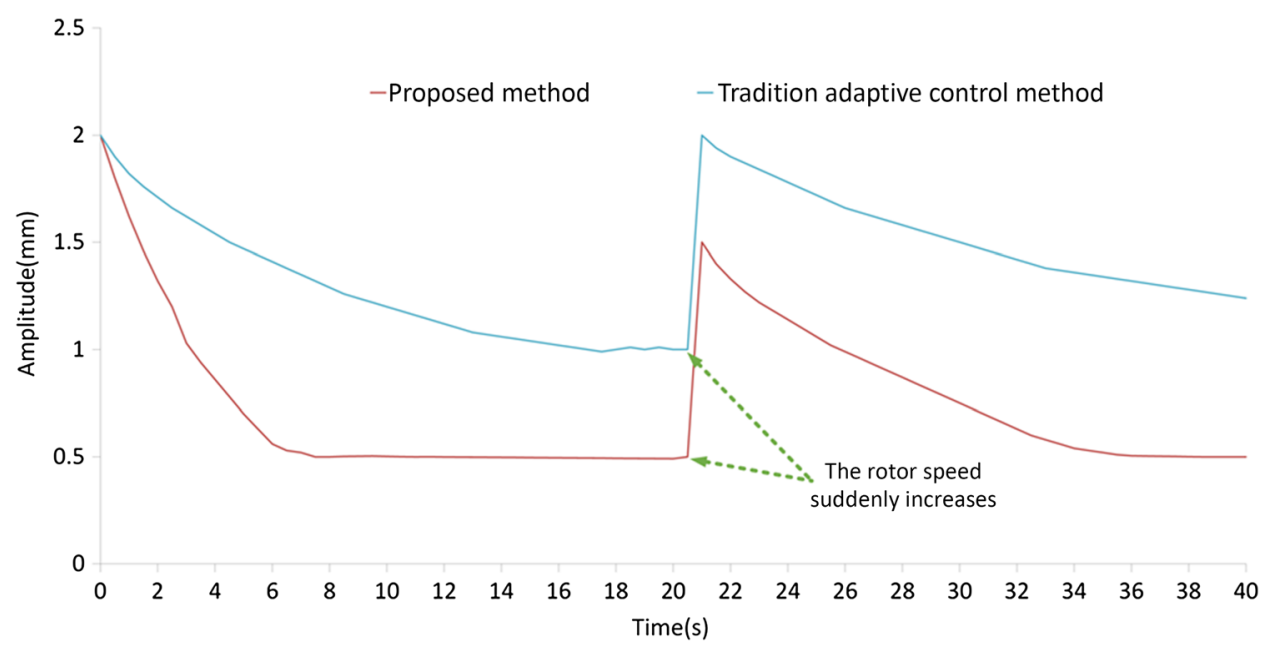

Fig. 11 Experimental comparison of two methods.

displacement sensor YXS-DWA). The vibration sensor is placed on the bearing surface to obtain the radial displacement, and the balancing head is placed at the end of the rotor to compensate for the unbalanced mass. The motor drives the spindle to rotate at different speed. The vibration sensor collects the vibration signal of the spindle and transmits the signal to the controller. The software part of the controller adopts the LQR algorithm proposed in this paper. The controller receives the vibration signal, calculates the balancing force $u(t)$ based on LQR control, and transmits the balancing force to the balancing head. Finally, the actuator outputs a balancing force to correct the imbalance and suppress the vibration.

In order to verify the balancing effect of the control method proposed in this paper, the online active balancing control method for time-delay rotor based on LQR control and the traditional adaptive control method were used in the experiment to carry out active balancing control on the rotor system, and the experiment results were compared. The initial rotational speed was set as $3000 \mathrm{rpm}$, and the initial vibration value was measured as $2 \mathrm{~mm}$. The experiment results are shown in Fig. 11.

As shown in Fig. 11, the initial vibration amount of the rotor system is $2 \mathrm{~mm}$. We applied the proposed method to the rotor dynamic balance system, then the vibration amplitude is reduced to $0.5 \mathrm{~mm}$ within $7.5 \mathrm{~s}$. However, when the traditional adaptive control method is adopted to the rotor dynamic balance system, the vibration amplitude is only reduced to $1.0 \mathrm{~mm}$ within $12.5 \mathrm{~s}$. When the rotor speed suddenly increases, the amplitude of the vibration also increases. The result shows that the proposed method can reduce the vibration to $0.52 \mathrm{~mm}$ within $13 \mathrm{~s}$ after the amplitude of the vibration increases to $1.53 \mathrm{~mm}$. On the contrary, the vibration amplitude is reduced to $1.35 \mathrm{~mm}$ within $20 \mathrm{~s}$ when the traditional adaptive control method is applied to the rotor dynamic balance system. Therefore, the proposed method in this paper can achieve better vibration control effects for the time-delay rotor system than that of the traditional adaptive control method, and the balancing speed is also faster.

\section{Conclusions}

In this paper, the active balancing control method for the time-delay rotor system is proposed. The dynamic model of the time-delay rotor system with time-delay is established, and the integral transformation term is introduced to transform the time-delay rotor system dynamics model into a dynamic model without time-delay. Then, according to the linear-quadratic optimal control theory, the active balancing control law of the time-delay rotor is designed. Moreover, the influence of weight matrix $Q$ and $R$ on the design of control law is discussed. Finally, to verify the correctness of the proposed method, the simulation model of the time-delay rotor control system is constructed in MATLAB. Meanwhile, we set up the experimental platform to carry out 
the experiments. The results show that the method can effectively suppress the vibration of the time-delay rotor system under different time-delay.

\section{Acknowledgments}

This research was funded by the National Key Research and Development Plan of China (No. 2018Y FB2000505), Nation Nature Science Foundation of China (No. 61806067), and the Key Research and Development Project of Anhui Province (No. 201904A06020024).

\section{References}

1. A. Shrivastava and A. R. Mohanty, "Identification of unbalance in a rotor system using a joint input-state estimation technique," J. Sound Vibr. 442(3), 414-427 (2019).

2. K. Ding et al., "Vibration investigation of rotor system with unbalance and blade-casing rubbing coupling faults," J. Vibroeng. 22(2), 353-365 (2020).

3. Z. C. Qiu, C. Li, and X. M. Zhang, "Experimental study on active vibration control for a kind of two-link flexible manipulator,” Mech. Syst. Signal Process. 118(March 1), 623-644 (2019).

4. W. Zhongbo, M. Chuan, and Z. Changsheng, "Current compensation control of multiple frequency vibrations of the rotor in active magnetic bearing high speed motors," Proc. CSEE 38(1), 275-284 (2018).

5. J. $\mathrm{Xu}$ and $\mathrm{X}$. Sun, "A multi-directional vibration isolator based on quasi-zero-stiffness structure and time-time-delay active control," Int. J. Mech. Sci. 100, 126-135 (2015).

6. F. Raufmehr and M. Rezaei, "Fuzzy logic-based scalable video rate control algorithm for high-delay applications of scalable high-efficiency video coding," J. Electron. Imaging 27(4), 043013 (2018).

7. S. Heindel, F. Becker, and S. Rinderknecht, "Unbalance and resonance elimination with active bearings on a Jeffcott rotor," Mech. Syst. Signal Process. 85(February), 339-353 (2017).

8. A. Mironchenko and F. Wirth, "Input-to-state stability of time-delay systems: criteria and open problems," in IEEE 56th Annu. Conf. Decis. and Control (CDC), Melbourne, pp. 3719-3724 (2017).

9. H. Zheng et al., "Shaft longitudinal vibration active control based on the adaptive method," J. Vibr. Shock 37(4), 203-207, 218 (2018).

10. N. A. Saeed and W. A. El-Ganaini, "Time-time-delay control to suppress the nonlinear vibrations of a horizontally suspended Jeffcott-time-delay rotor system," Appl. Math. Modell. 44(April), 523-539 (2017).

11. L. Wang et al., "Hybrid time-variant reliability estimation for active control structures under aleatory and epistemic uncertainties," J. Sound Vibr. 419, 469-492 (2018).

12. H. L. Hu and L. D. He, "Control of critical speed vibrations of a single-span rotor by a rotor dynamic vibration absorber at different installation positions," J. Mech. Sci. Technol. 31(5), 2075-2081 (2017).

13. D. S. Huang, J. Q. Zhang, and Y. L. Liu, "The PID semi-active vibration control on nonlinear suspension system with time delay," Int. J. Intell. Transp. Syst. Res. 16(7), 1-13 (2017).

14. J. Zhang et al., "Robust model predictive control for uncertain positive time-delay systems," Int. J. Control Autom. Syst. 17(2), 307-318 (2019).

15. H. Li, G. Xu, and G. Xu, "Mechanical vibration monitoring system based on wireless sensor network," Int. J. Online Eng. 14(6), 126-137 (2018).

16. Z. Wang and C. M. Mak, "Application of a movable active vibration control system on a floating raft," J. Sound Vibr. 414, 233-244 (2018).

17. S. Hongxin et al., "Time delay compensation for the active cable vibration control using giant magnetostrictive actuators," J. Vibr. Shock 36(14), 208-215 (2017).

18. W. H. Kwon and A. E. Pearson, "Feedback stabilization of linear systems with time-delay control," IEEE Trans. Autom. Control 25(2), 266-269 (1980). 
19. H. Im, H. H. Yoo, and J. Chung, "Dynamic analysis of a BLDC motor with mechanical and electromagnetic interaction due to air gap variation," J. Sound Vibr. 330(8), 1680-1691 (2011).

20. B. Brekhna et al., "Robustness analysis of superpixel algorithms to image blur, additive Gaussian noise, and impulse noise," J. Electron. Imaging 26(6), 061604 (2017).

Juan Xu received her PhD from Hefei University of Technology, Hefei, China, in 2012. Currently, she works at Hefei University of Technology. Her current research interests include vibration active control and intelligent fault diagnosis.

Yang Zhao received his BS degree in mechanical engineering from Xuzhou University of Technology in 2014. He received his MS degree in mechanical engineering from Hefei University of Technology in 2019. His research interests include electrical control, vibration active control, and bearing dynamics analysis.

Zhanfeng Xu, graduated from Zhejiang University of Science and Technology in 2019. He is a graduate student majoring in mechanical and electronic engineering at Hefei University of Technology. He is mainly engaged in the research of vibration active control and intelligent fault diagnosis.

Benhong Zhang received his PhD from Hefei University of Technology, Hefei, China, in 2010. Currently, he is an associate professor and master supervisor of the School of Computer and Information, Hefei University of Technology. He is mainly engaged in vibration active control and distributed control. 